\title{
Human Interaction in a Virtual Reality Environment
}

\author{
Juan D. PELAEZ-RESTREPO ${ }^{1}$, Toh Yen PANG and Cees BIL \\ School of Engineering, RMIT University, Melbourne, Australia
}

\begin{abstract}
Virtual Reality (VR) and Augmented Reality (AR) are becoming useful tools to provide a visual representation of a product, a design or environment without the cost of building a prototype. Other applications include maintenance support, procedures development and workstation design. Currently, VR and AR systems can only display visual and audio cues. For example, in workstation design, i.e., a ship's bridge, aircraft flight deck, command centre, maintenance facility, etc., it is important to for design engineering to determine if the human operator can reach the gauges, switches and controls. To meet the ergonomic and human factor design criteria, the human operator must easily interact with all the displays, instruments, equipment, and controls. This impacts the design and location of seats, instrument panels and windows. VR and AR devices, such as the controllers and gloves, can track the position of the human hand and fingers in a virtual environment but are not able to detect realistic distance or interference with a virtual object. This paper presents a new VR prototype to allow an operator in a fully immersive environment to reach and touch any object in the virtual world. As a case, study the environment was applied to the cabin design of a medical helicopter. Feedback and suggestions from the medical personnel were obtained and are presented.
\end{abstract}

Keywords. Virtual/Augmented reality, workstation design, transdisciplinary design, helicopter cabin design, air mobile stroke unit

\section{Introduction}

Environments where people operate or interact with each other are difficult to design with traditional CAD tools as these do not capture or represent human ergonomic features and behaviours such as interference, human-machine and inter-human interaction, etc. Interactive and immersive technologies such as Virtual Reality (VR) and Augmented Reality (AR) are becoming useful tools to provide a visual representation of a product, design, or environment without the relative expensive cost of fabricating a prototype. Other VR/AR applications include maintenance support, procedures development and workstation design. Even with some limitations such as computational cost or motion sickness, VR/AR have become useful tools in the design of workspace and architectural projects.

In workspace design, e.g. a ship's bridge, command centre, maintenance centre, etc., it is important to determine if a human operator can reach the gauges, switches, and controls. The ergonomic design of a workspace requires an understanding of the variability in human bodies, their personal preferences, and the environment they interact

\footnotetext{
${ }^{1}$ Corresponding Author, Mail: juan.pelaez.restrepo@rmit.edu.au.
} 
with. All these will impact the design and location of seats, instrument panels and windows, and influence an operator's interaction with all the displays, instruments, and controls. For this reason, reach and touch capability in VR and AR is essential to understand the ergonomic and human factor when designing a workstation.

In this project, we use VR and AR technologies to facilitate human-workspace interactions and to improve the overall efficacy and effectiveness of such interactions. The tracking sensors and novel software algorithm was developed to detect the distance of a human hand to any point in the virtual world, and to provide feedback to the VR operator that contact has been made. The VR environment was used as part of the design process of the Air Mobile Stroke Unit (Air MSU), which is an ambulance helicopter to be used as response to emergencies in regional and remote areas [1].

\section{Background}

\subsection{VR Applied to Workstation Design}

Different studies have showed that VR can be used as a tool in the design of workstations and for training purposes [2]. More specifically, it can be used in the ergonomic design of workstations, where a virtual environment can be easily tested and modified than a physical prototype $[3,4]$.

Additionally, workplaces can be simulated for training purposes, in particular those with manual tasks and procedures that must be executed on a regular basis. VR presents a useful tool to reproduce a virtual representation of the workplace where the user can refine their skills before executing the task in the real world [5 - 8]. In the aerospace industry, some studies have been conducted to evaluate the design of luxury cabins using VR for business flights [10].

\subsection{Interaction in VR Environments}

At the current state of VR technology, one of the most challenging problems is to create a realistic environment in which the user can have the ability to reach and touch the objects in virtual environment [11,12]. Haptic technology, also known as kinaesthetic

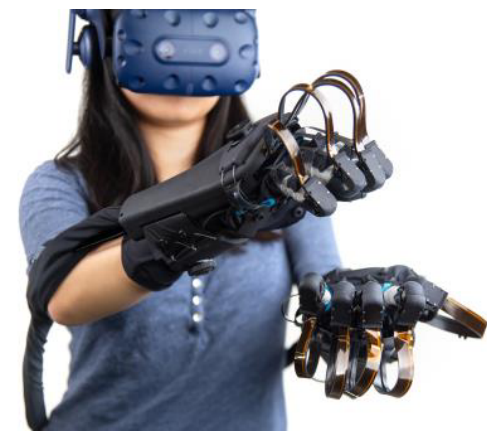

Figure 1. Haptic gloves (www.haptx.com). communication or $3 \mathrm{D}$ touch, refers to any technology that can create an experience of touch by applying forces, vibrations, or motions to the user [13]. Most advanced forms of haptic feedback are gloves that allow the user to grab an object and move it. Force-feedback option can be added by using actuators or air pressure in the fingers. This type of advanced haptic technology is still in development and current gloves are typically large and heavy (Figure 1). However, the type of haptic feedback needed depends on the application. For some applications, it is sufficient to be able to touch the object. Infrared hand tracking devices can detect the position of the user's hands relative to external tracking sensors. 
The position of the hands in the real world can then be translated to a position in the virtual world [11]. The next development is to implement a technique get the desired interaction between the virtual hands and the components of the VR environment $[12,14]$.

This paper describes a technique for determining the distance between the hands and any part of the virtual world and to detect if the hands are touching the object. When an object is touched it is highlighted (visual cue) and the hand-held controllers vibrate (sensory cue). This capability is very useful and sufficient in the design of workspaces where the ability to easily reach and touch instruments, controls and other objects must be verified.

\subsection{Determining Perceived Distance}

In the past decade, many design engineers have used VR to design immersive environments, particularly in large scale projects, because VR provides them the ability to create a low-cost iterative design tool for testing and prototyping true scale $3 \mathrm{D}$ products or systems.

In commercial product developments, VR is being used as a tool not just for visualization but also for develop the manufacturing workflows and ergonomic production workstations. However, one of the challenges to develop high fidelity VR environment is to reproduce a true to scale perception for the users. Some studies have suggested that distances appear to be compressed in immersive virtual environments when compared to the real world. Hence, many studies have been conducted to understand the depth, distance perception, and the visual characteristics of the virtual representation of the real world. For example, Loomis et al. have investigated, through experimental study of perceived space and motor action, the process of mapping between real space with its corresponding virtual space $[15,16]$. However, the reason for this disagreement in the perception is yet unidentified $[5,17]$.

\subsection{Transdisciplinary Design}

This project is transdisciplinary in the sense that the key concepts, expertise, and methods are drawn from multiple disciplines, such as engineering, technology, human factor, computer science and information visualization. In line with this, we utilize the engineering knowledge to develop the 3D models, the emergent VR technology (HTC Vive) to manipulate the $3 \mathrm{D}$ virtual object, the human factor approach to evaluate the interactions between real and virtual environment, and the $3 \mathrm{D}$ modelling software to visualize and evaluated results.

\section{VR Equipment and Application Development Tools}

For the development of the virtual Reality environment the game engine Unity (www.unity.com) was used due to its embedded tools and libraries that make the implementation of VR functions a straightforward process. A process diagram of the development is described in Figure 2. 


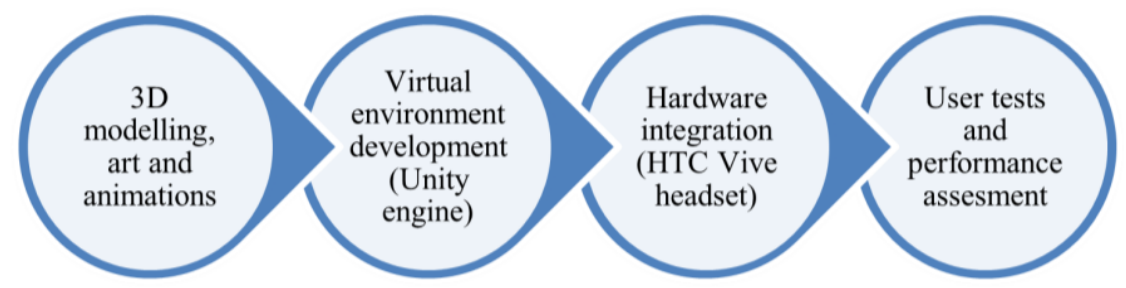

Figure 2. Development process diagram.

The work on the VR application has been focused on two fronts, first to implement interactions with 3D models in a virtual environment, and second to use these functions in an ambulance helicopter virtual model, in which a user could interact with the aircraft model and its parts.

\section{1. $3 D$ Modelling}

All the 3D objects were modelled using the computer-aided design software, i.e. 3DEXPERIENCE as shown in Figures 3-5. The virtual models consisted of an ambulance helicopter, a stretcher, a human mannequin, crew seats, and a CT-scanner unit.

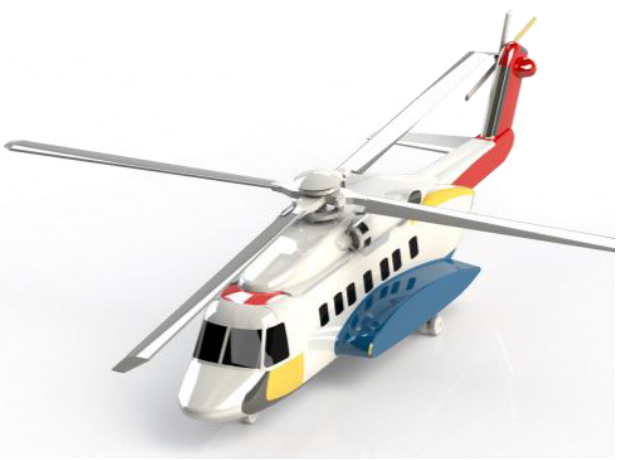

Figure 3. Helicopter CAD model.

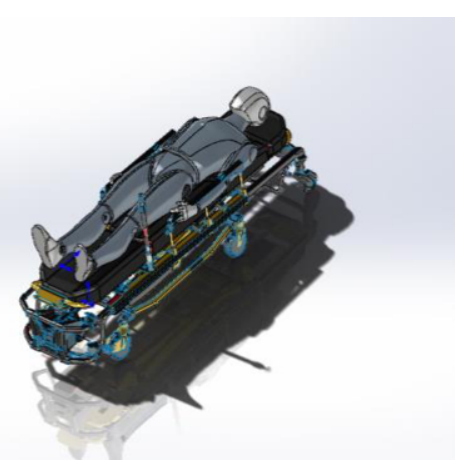

Figure 4. Patient + stretcher $3 \mathrm{D}$ model.

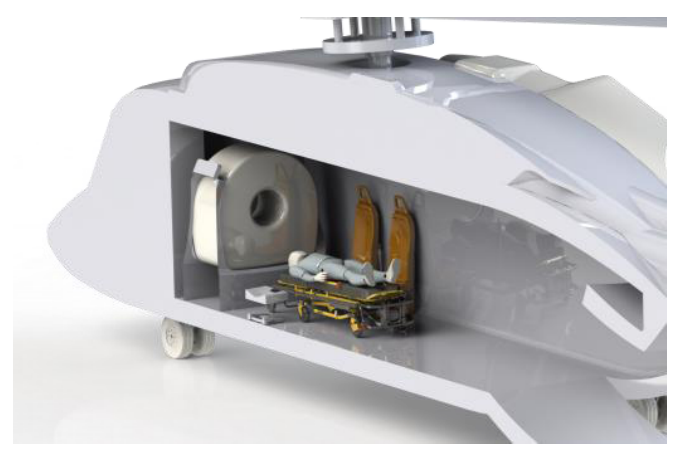

Figure 5. Ambulance Helicopter Virtual Assembly 


\section{VR environment development}

One of the main goals for the development of the VR application was to create a reach and touch capability in the virtual environment. This will contribute to the humancentered design of an efficient and ergonomic workspace, such as a ship's bridge, aircraft flight deck, operator station, medical unit, etc.

The team was composed of a transdisciplinary group of medical professionals, biomedical, mechanical, and aerospace engineers that met regularly to identify requirements and discuss progress. The virtual reality application was developed so the user can experience and evaluate the layout of equipment and interact with different components inside and outside the aircraft.

This paper is reporting on the conceptual phase of the study, which included the simplified models of the aircraft and its components. We intend to use the results collected during the pilot experiment to refine and further develop specific components to fulfil the user requirements.

The pilot experiment was conducted with a professional health worker to seek her feedback through semi-structured interview session. We sought to understand the subjective measurements of performance, usability, and comfort of the VR application.

\subsection{VR Equipment}

An HTC Vive PRO headset and controllers were used in the development of the virtual reality application (Figure 6). This headset uses room scale tracking technology, which allows the user to operate in a $5 \mathrm{~m} \mathrm{x}$ $5 \mathrm{~m}$ area. The user uses two hand-held controllers to interact with the virtual environment. The position is determined using a minimum of two fixed infra-red tracking stations.

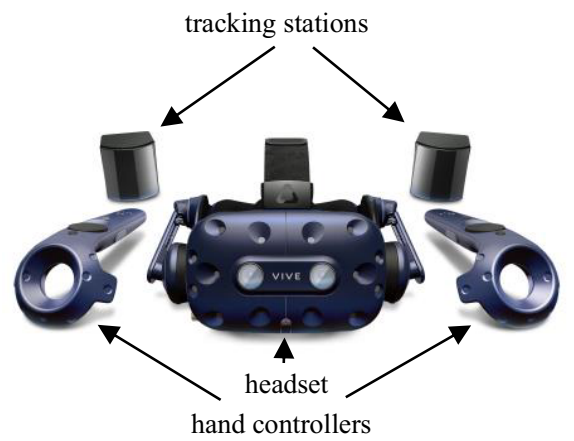

Figure 6. HTC Vive Pro system.

\subsection{Reach and Touch in VR Environment}

The VR application for the ambulance helicopter developed in this project can be used as a virtual experience inside Unity engine, which allows users to test all the
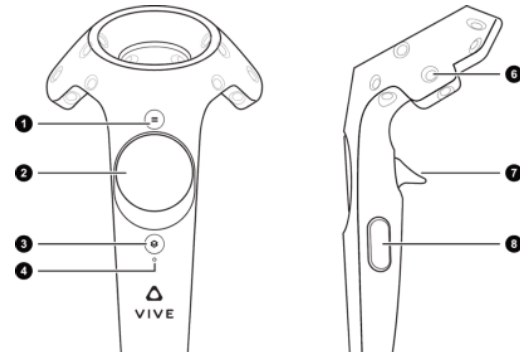

Figure 7. Hand controllers. functionalities of the application and perform changes or corrections as needed.

The main interaction tool while immersing in the virtual environment was the HTC Headset. Additionally, the users could use two controllers to interact with the 3D models inside the virtual environment. The controller appeared as a pair of gloves, which enable users to have a sense of realism. Users could press the buttons on the controllers to perform the basic operations, such as touch, grab, etc. as described in Figure 7. 
- Button 1: access setup menu

- Button 2: allows the user to tele transport to any part of the scene that cannot be reached by walking in the "real world"

- Button 8: allows the user to grab any object inside the virtual world.

- Button 7: allows the user to interact with the menu elements or with the models of the virtual world.

\subsection{User-Defined VR Interaction}

Using Unity engine, a virtual environment was developed, in which a scene trying to resemble an Australian outback landscape was built. In this scene the 3D models previously developed were included in addition to the terrain, lights, and textures. A screenshot of the scene is shown in Figure 8.
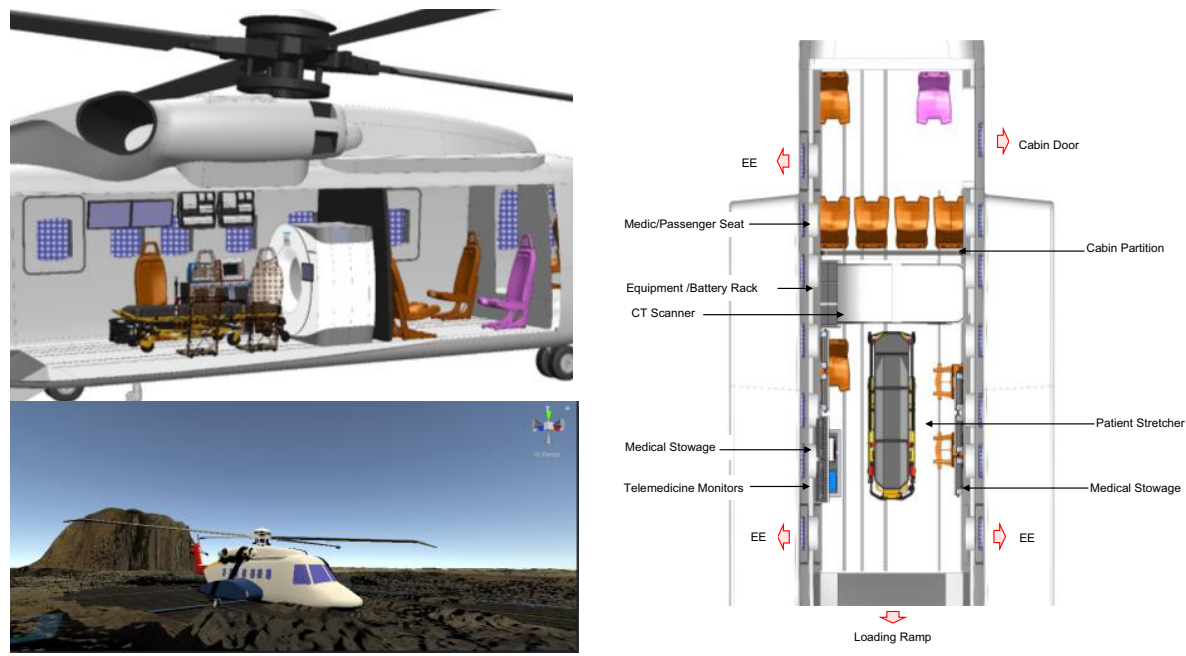

Figure 8. Virtual environment scene.

To achieve interactive actions like grab, touch, launch with the 3D models in the virtual environment, scripts available in the Unity engine library were used. The library was modified to achieve the behaviour relevant for the project such as show or hide controls. Several scripts where modified or newly written to get the desired behaviour of the virtual character inside the virtual environment.

When an object or any part of the virtual environment is touched, the object is highlighted with a coloured outline (visual) and the controllers vibrate to provide haptic feedback. Figure 9 shows images from virtual environment when using the controllers with reach and touch capability. Touch objects are highlighted with a yellow outline. 

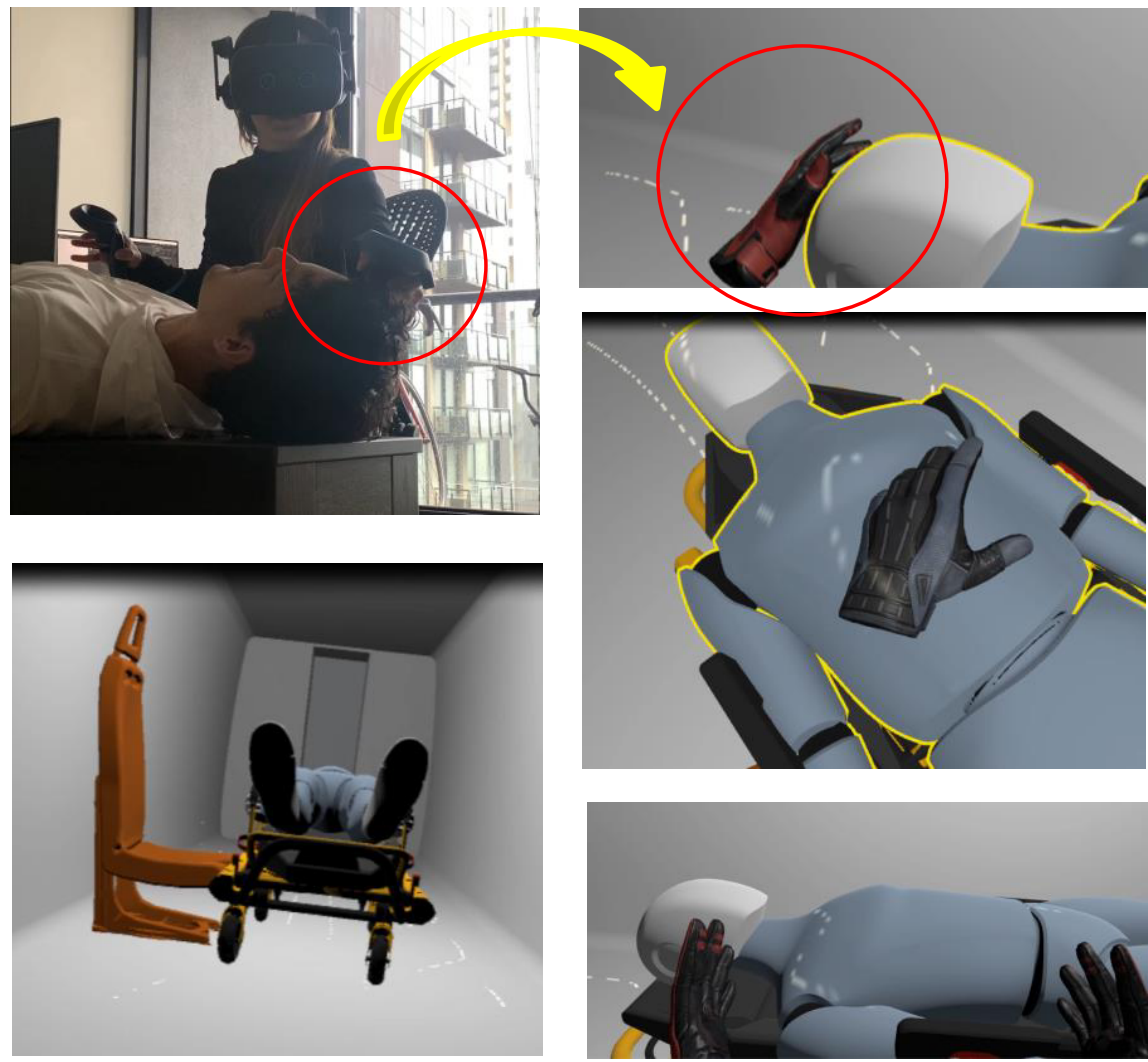

Figure 9. Interaction system sample.

The application is being built trying to simulate the scenario a team member of the Ambulance would have in case of a person needs attention. That is why a human dummy is shown in the figures above.

\section{Medical Helicopter Cabin Design - Case Study}

To investigate the effectiveness of research and touch capability in a VR environment, a model of a cabin of a medical helicopter was created with seats, stretcher, and a model patient. To test whether the interaction performance in the virtual environment represented accurately an ambulance scenario, a professional radiographer was invited to participate in an experimental setup and to provide feedback on perceived distance and interactions (Figure 10).

\subsection{Apparatus and participants}

For this case study a medical professional was invited to use the VR environment with modelled seat, stretcher and patient located inside a helicopter cabin. Various medical 
procedures were simulated, and feedback was obtained on realism, interaction experience and overall effectiveness.

As a new user of VR technologies, her opinions about the application performance and realism are very useful from the usefulness as a design and training tool point of view.

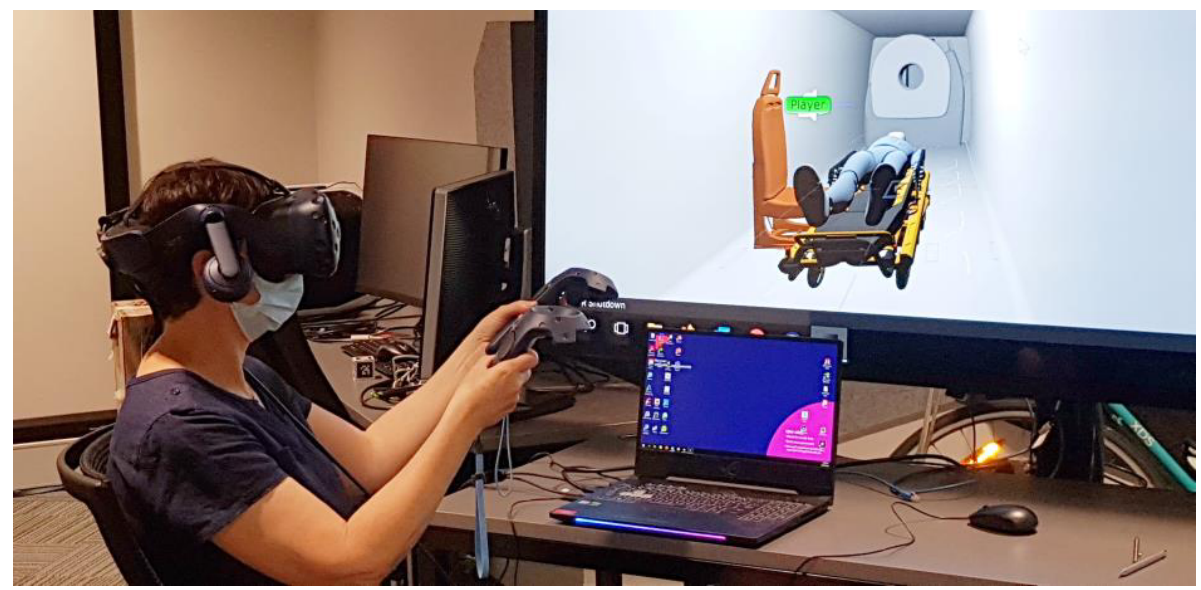

Figure 10. VR Environment Test Setup.

The test was performed at the RMIT VXLab (www.rmit.edu.au), using the equipment described in section 2.1. The virtual model consisted of a helicopter cabin with a CT-scanner and a stretcher with a modelled patient. Also in the scene was a seat next to the stretcher and a real seat was placed in the same location for the participant to sit on during the experiment. The participant was also asked to stand up and walkaround the stretcher. After giving some basic instructions on how to use the VR application, the participant was asked questions about how she perceived the distance and shapes of the $3 \mathrm{D}$ objects, the interaction with objects surfaces with the virtual environment.

\subsection{Results}

Overall, the participant was satisfied with the VR environment and the ability to interact with various objects and how real the experience of interacting with the virtual patient was, it was possible to "cradle the patient's head". Also, the participant said that the perception of the patient and stretcher sizes and distances felt very accurate. Additionally, it was also expressed that during the test there was no feeling of dizziness or any discomfort produced by the VR environment.

The lack of any force feedback while interacting with objects in the virtual environment was mentioned as a downside and would be noticeable in case of, for example, administering CPR. The participant also suggested to include other persons, e.g. co-workers, in the same environment to make collaboration and teamwork more realistic. In any outcall, there are five medical and support staff onboard and people need to manoeuvre around co-workers within a confined space.

Another comment was about the perceived distance of the CT-scan unit. The participant felt that the interior space of the helicopter seemed a lot bigger that the real 
world and expressed that object at a distance in the virtual environment seemed "very far away". Although the scale and dimensions of the helicopter cabin were correctly modelled the depth perception error seems more an issue with how the environment is displayed in the headset.

\section{Conclusions}

A basic reach and touch capability for a virtual environment was developed to support the design of workspaces where a human operator must interact with objects in the virtual world. The only device needed are standard hand controllers that are common accessories to a VR or AR device. These hand controllers can detect hand position, but is not able to interact with the environment, such as touching components, and the aim of this project is to develop the capability to use standard hand controllers to reach and touch objects in the virtual environment. More sophisticated and expensive haptic gloves are not always required for ergonomic workplace design where force feedback, etc. are not essential.

As a case study a cabin of a medical helicopter was designed to determine that a medical professional can adequately attend to the patient in a confined space. A medical professional was invited to use the virtual environment setup and provide feedback on realism, interaction experience and general effectiveness. The general observations were:

- The virtual environment gives the user a good experience while interacting with different surfaces and shapes and provided an accurate feeling of size and distance.

- To improve the feeling of realism and the usability as a design and training tool, it is needed to find a way to give the user a more realistic feeling of the surroundings and even force haptic feedback, for example in case of CPR.

- To get a more realistic training environment is important to create a "multi-user" environment in which the teamwork procedures can be reproduced accurately.

Feedback given during this case study will be used to improve the application aiming to develop a tool useful for aircraft design purposes, to improve the immersion experience for the user, a more detailed cabin interior is going to be modelled using CAD and 3D modelling software.

Future work will focus on the development a more realistic virtual working environment the interior space of the helicopter cabin. Additional trackers will be included in the environment, so the user will wear those in different body parts and then get feedback while in the virtual world if they are contacting the virtual surfaces. Finally, a multi-user environment will be developed allow multiple persons including hearing guidance of how to use the VR application and some common procedures developed in ambulances will be reproduced as a training exercise.

\section{Acknowledgement}

defence science institute

The authors acknowledge the collaborative research grant from the Defence Science Institute (DSI) for the development of this project 


\section{References}

[1] D. Chipperfield, M. Cheesman, C. Bil, G. Hanlon, Transdisciplinary Design Aspects of an Air Mobile Stroke Unit, Advances in Transdisciplinary Engineering, 2020, Vol. 12, pp. 250-259.

[3] P. Budziszewski, A. Grabowski, M. Milanowicz, and J. Jankowski, Workstations for people with disabilities: an example of a virtual reality approach, Int J Occup Saf Ergon, 2016, Vol. 22, No. 3, pp. 367-73, doi: 10.1080/10803548.2015.1131069.

[4] H. Nguyen, C. Pontonnier, S. Hilt, T. Duval, and G. Dumont, VR-based operating modes and metaphors for collaborative ergonomic design of industrial workstations, Journal on Multimodal User Interfaces, 2016, Vol. 11, No. 1, pp. 97-111, doi: 10.1007/s12193-016-0231-x.

[5] C. Pontonnier, G. Dumont, A. Samani, P. Madeleine, and M. Badawi, Designing and evaluating a workstation in real and virtual environment: toward virtual reality based ergonomic design sessions, Journal on Multimodal User Interfaces, 2013, Vol. 8, No. 2, pp. 199-208, doi: 10.1007/s12193013-0138-8.

[6] F. Caputo, A. Greco, E. D'Amato, I. Notaro, and S. Spada, On the use of Virtual Reality for a human-centered workplace design, Procedia Structural Integrity, 2018, Vol. 8, pp. 297-308, doi: https://doi.org/10.1016/j.prostr.2017.12.031.

[7] Y. M. Anoffo, E. Aymerich, and D. Medda, Virtual Reality Experience for Interior Design Engineering Applications, 26th Telecommunications Forum (TELFOR), 20-21 Nov. 2018,, pp. 1-4, doi: 10.1109/TELFOR.2018.8612026.

[8] H. Lee and W. C. Cha, Virtual Reality-Based Ergonomic Modeling and Evaluation Framework for Nuclear Power Plant Operation and Control, Sustainability, 2019, Vol. 11, No. 9, doi: 10.3390/su11092630.

[9] S. Roberts, R. Page and M. Richardson, Designing in virtual environments: The integration of virtual reality tools into industrial design research and education, DRS 2020: Synergy, 2020: Design Research Society, pp. 1628-1643.

[10] F. De Crescenzio, S. Bagassi, S. Asfaux, and N. Lawson, Human centred design and evaluation of cabin interiors for business jet aircraft in virtual reality, International Journal on Interactive Design and Manufacturing (IJIDeM), 2019, Vol. 13, No. 2, pp. 761-772, doi: 10.1007/s12008-019-005658 .

[11] H. Tilak and R. Cheng-Yue, Egocentric Hand Tracking for Virtual Reality. Stanford EE 267: Virtual Reality, Corpus ID: 173174646, 2016.

[12] J. Lin and J. P. Schulze, Towards naturally grabbing and moving objects in VR, Electronic Imaging, 2016, No. 4, pp. 1-6.

[13] H. Culbertson, S.B. Schorr, A.M. Okamura, Haptics: The Present and Future of Artificial Touch Sensations, Annual Review of Control Robotics and Autonomous Systems, 2018, Vol. 1, pp. $385-$ 409.

[14] C. Fang, Y. Zhang, M. Dworman, and C. Harrison, Wireality: Enabling Complex Tangible Geometries in Virtual Reality with Worn Multi-String Haptics, Proceedings of the $2020 \mathrm{CHI}$ Conference on Human Factors in Computing Systems, 2020, pp. 1-10.

[15] J. Loomis \& J. Knapp, Visual perception of egocentric distance in real and virtual environments, In L. J. Hettinger \& M. W. Haas (Eds.), Virtual and adaptive environments: Applications, implications, and human performance issues Lawrence Erlbaum Associates Publishers, 2003, pp. 21-46. https://doi.org/10.1201/9781410608888.pt1.

[16] J.M Loomis JM, Da Silva JA, Philbeck JW, Fukusima SS. Visual Perception of Location and Distance. Current Directions in Psychological Science, 1996;5(3), pp. 72-77. doi:10.1111/14678721.ep10772783.

[17] V. Interrante, B. Ries and L. Anderson, Distance perception in immersive virtual environments, revisited, IEEE virtual reality conference (VR 2006), 2006, pp. 3-10. 